\title{
Orientação às lactantes acerca do aleitamento materno frente à pandemia COVID-19
}

\author{
Guidance on breastfeeding during COVID-19 pandemic \\ Orientación a las lactantes respecto a la lactancia materna frente la \\ pandemia COVID-19 \\ Ana Pedrina Freitas Mascarenhas ${ }^{1}$, Klessiane Mendes de Fontes² ${ }^{2}$ Thalys \\ Maynnard Costa Ferreira ${ }^{3}$, Aldaires Peixoto da Silva ${ }^{4}$, Juliana Maria Azevedo \\ Pessoa da Silva ${ }^{5}$

\footnotetext{
${ }^{1}$ Enfermeira. Universidade Federal da Paraíba. João Pessoa, Paraíba

${ }^{2}$ Enfermeira. Universidade Federal da Paraíba. João Pessoa, Paraíba

${ }^{3}$ Docente do Centro Universitário de João Pessoa. João Pessoa, Paraíba

${ }^{4}$ Enfermeira. Hospital Belarmino Correia. Goiana, Pernambuco

${ }^{5}$ Enfermeira. Universidade Federal do Pernambuco. Recife, Pernambuco
}

\section{RESUMO}

Frente à pandemia Covid-19, é essencial identificar orientações feitas por órgãos oficiais sobre aleitamento materno. Estudo documental com abordagem quantitativa, objetivando fazer levantamento dos documentos oficiais para orientações e recomendações sobre aleitamento materno durante a pandemia, sumarizando instruções para viabilizar a prática de aleitamento materno seguro. Corpus documental composto por 8 documentos emitidos pelo Ministério da Saúde e Sociedade Brasileira de Pediatria, indexados na BVS, operacionalizado mediante

Autor de Correspondência:

*Ana Pedrina Freitas Mascarenhas. E-mail: anapedrinajp@hotmail.com 
busca eletrônica de artigos pelos descritores Pandemias, Aleitamento Materno, Infecções por Coronavírus separados por operador booleano "AND". Dados analisados delinearam quatro categorias de orientações: parturiente assintomática, parturiente sintomática, mulheres com diagnóstico de infecção por novo Coronavírus e orientações sobre bancos de leite. As publicações são importantes instrumentos de apoio ao profissional de saúde, sendo imprescindível que este esteja mantendo constante e pertinente atualização sobre temática do cuidado à gestante, puérpera e aleitamento materno na pandemia existente.

Palavras-chave: Pandemias. Aleitamento Materno. Infecções por Coronavírus.

\begin{abstract}
During Covid-19 pandemic, it is essential to identify the guidelines provided by official agencies on breastfeeding. This is a documentary study with a quantitative approach, aiming to make a survey of the official documents for guidance and recommendations on breastfeeding during the pandemic, summarizing instructions to allow safe breastfeeding practice. This is a documentary corpus consisting of 8 documents issued by the Ministry of Health and the Brazilian Society of Pediatrics, indexed in the VHL, made operational by electronic search of articles with the descriptors Pandemics, Breastfeeding, Coronavirus Infections, separated by Boolean operator "AND". Analyzed data outlined four categories of guidelines: asymptomatic parturient, symptomatic parturient, women diagnosed with new Coronavirus infection, and guidance on milk banks. Publications are an important support tool for health professionals, and it is essential that they keep constant and pertinent update on the theme of care for pregnant women, puerperal women, and breastfeeding in the existing pandemic.
\end{abstract}

Keywords: Pandemics. Breast Feeding. Coronavirus Infections.

\title{
RESUMEN
}

Ante la pandemia de Covid-19, es esencial identificar las pautas proporcionadas por las agencias oficiales sobre lactancia materna. Estudio documental con un enfoque cuantitativo, que tiene por objetivo evaluar la orientación sobre la lactancia materna durante una pandemia, resumiendo las instrucciones para la práctica segura de la lactancia materna. Cuerpo documental compuesto por 8 documentos del Ministerio de Salud y la Sociedad Brasileña de Pediatría, indexados en la BVS, operados mediante búsqueda electrónica por los descriptores Pandemias, Lactancia, Infecciones por Coronavirus aisladas por el operador booleano "Y". Los datos analizados describieron cuatro categorías de pautas: parturienta asintomática y sintomática, mujeres diagnosticadas con nueva infección por Coronavirus y orientación sobre bancos de leche. Las publicaciones son herramientas importantes para apoyar a los profesionales de la salud, y es esencial que se mantengan actualizadas sobre la atención a las mujeres embarazadas, las mujeres puerperales y la lactancia materna en la pandemia existente.

Palabras clave: Pandemias. Lactancia Materna. Infecciones por Coronavirus. 


\section{INTRODUÇÃO}

Em novembro de 2019, um surto de doença respiratória, causado pelo novo coronavírus (SARSCoV-2), foi detectado na cidade de Wuhan, na China. Em dois meses foram confirmados milhares de casos de Covid-19, que resultaram em inúmeros óbitos. Em março de 2020, o novo coronavírus disseminouse para mais países, continuando a causar doença respiratória e óbitos, especialmente em grupos de risco como idosos, gestantes, imunodeprimidos e outros ${ }^{1}$.

No Brasil, o primeiro caso detectado foi um homem brasileiro de 61 anos, que viajou a trabalho para Itália, onde ficou de 9 a 21 de fevereiro, assintomático quando retornou para a cidade de São Paulo. Entretanto dois dias depois apresentou sintomas e a confirmação oficial veio do Instituto Adolfo Lutz, sendo o primeiro caso da doença confirmado em toda a América Latina ${ }^{2}$. Até o presente momento, o Brasil apresenta 330.890 casos confirmados da doença, com 21.048 óbitos e taxa de letalidade de $6,4 \%{ }^{3}$.

O Covid-19 foi considerado um parente da síndrome respiratória aguda grave (SARS) e, até o momento, a fonte do SARS-CoV-2 permanece desconhecida. No entanto, a infecção por SARS-CoV-2 foi associada ao contato com um vendedor local de frutos do mar em Wuhan que vendeu ilegalmente alguns animais da vida selvagem, incluindo morcegos ${ }^{4}$.

Dentre as condições e fatores de risco a serem considerados para possíveis complicações pelo Covid-19 estão grávidas em qualquer idade gestacional, puérperas até duas semanas após o parto (incluindo as que tiveram aborto ou perda fetal) e crianças menores de 5 anos (sendo que o maior risco de hospitalização é em menores de 2 anos, especialmente as menores de 6 meses com maior taxa de mortalidade); população indígena aldeada ou com dificuldade de acesso, indivíduos menores de 19 anos de idade em uso prolongado de ácido acetilsalicílico (risco de síndrome de Reye); indivíduos que apresentem: pneumopatias (incluindo asma), tuberculose, cardiovasculopatias, nefropatias, hepatopatias, doenças hematológicas, distúrbios metabólicos (incluindo diabetes mellitus), transtornos neurológicos e do desenvolvimento que podem comprometer a função respiratória ou aumentar o risco de aspiração (disfunção cognitiva, lesão medular, epilepsia, paralisia cerebral, síndrome de Down, acidente vascular encefálico - AVE ou doenças neuromusculares); imunossupressão associada a medicamentos (corticóide, quimioterápicos, inibidores de TNF-alfa), neoplasias, HIV/aids ou outros, e obesidade (especialmente aqueles com índice de massa corporal (IMC) $\geq 40$ em adultos) $)^{5}$.

O aleitamento materno é a pedra angular da sobrevivência, nutrição e desenvolvimento infantil e saúde materna. A Organização Mundial de Saúde (OMS) e o Fundo das Nações Unidas para a Infância (UNICEF) recomendam que a amamentação seja iniciada na primeira hora após o nascimento, ofertada exclusivamente nos primeiros 6 meses de vida e continuada, com alimentos complementares seguros e adequados, até 2 anos ou mais 6 .

O leite materno é o único alimento que assegura nutrientes em qualidade e quantidade ideais para o lactente e o aleitamento materno permite, ainda, o vínculo, afeto, proteção entre o binômio mãe-filho, promovendo não só benefícios para a lactente, mas também para a mãe como a prevenção de câncer de mama.

Assim, o aleitamento é a estratégia que mais previne mortes infantis, além de promover a plenitude do desenvolvimento físico e mental dos primeiros anos e, a longo prazo, a redução de doenças que se manifestam na vida adulta, como processos crônicos ${ }^{7}$.

O contato pele a pele, além de favorecer o aleitamento materno precoce e o desenvolvimento do vínculo mãe e filho, propicia níveis favoráveis de batimentos cardíacos e respiração da criança, diminuindo o choro, conferindo conforto ao recém-nascido ${ }^{8}$. 
As gestantes, puérperas e crianças menores de 05 anos, enquadram-se no grupo de risco, pois são mais vulneráveis a infecções. Embora ainda não tenha estudo específico conclusivo, esse vírus apresenta letalidade associado à história clínica de comorbidades nesses grupos. Portanto, os cuidados com gestantes e puérperas devem ser rigorosos e contínuos, independentemente do histórico clínico das pacientes, requerendo uma atenção mais específica diante do cenário de pandemia. ${ }^{5}$

Diante do exposto, estabeleceu-se a seguinte pergunta norteadora: "Quais são as orientações na promoção do aleitamento materno frente à pandemia pelo Coronavírus?" Como resposta à essa pergunta, este estudo tem como objetivo fazer um levantamento dos documentos oficiais que trazem orientações e recomendações sobre o aleitamento materno no momento de pandemia, como também sumarizar essas instruções para viabilizar a prática do aleitamento materno seguro.

\section{MÉTODO}

Estudo do tipo documental com abordagem quantitativa, que trilha caminhos semelhantes à pesquisa bibliográfica, recorrendo, entretanto, a fontes primárias, que não foram ainda tratadas, mais diversificadas e dispersas, tais como: tabelas estatísticas, jornais, revistas, relatórios, documentos oficiais, cartas, filmes, fotografias, pinturas, tapeçarias, relatórios de empresas, vídeos de programas de televisão, etc ${ }^{9}$.

Gil $^{10}$ evidencia que a pesquisa documental compreende as fases: determinação dos objetivos; elaboração do plano de trabalho; identificação das fontes; localização das fontes e obtenção do material; tratamento dos dados; confecção das fichas e redação do trabalho; construção lógica e redação do trabalho. Essas fases ocorrem numa sequência natural e de forma articulada.

O corpus documental foi composto pelos atos normativos oficiais do tipo Nota Técnica e Nota de Alerta de autoria do Ministério da Saúde e da Sociedade Brasileira de Pediatria do ano 2020, indexados na base de dados Biblioteca Virtual em Saúde de forma íntegra e gratuita, operacionalizado mediante busca eletrônica de artigos pelos descritores pandemias, aleitamento materno, infecções por coronavírus separados por operador booleano "AND". Excluíram-se as notas revogadas, duplicadas ou que não atendessem ao objetivo do estudo. A busca dos documentos ocorreu no mês de abril de 2020, totalizando 03 Notas de Alerta da Sociedade Brasileira de Pediatria e 05 Notas Técnicas do Ministério da Saúde, totalizando 08 documentos que atenderam ao estudo. Apenas 01 nota não atendia ao estudo, tendo sido descartada.

A análise documental inicia-se pela avaliação preliminar de cada documento, realizando o exame e a crítica do mesmo, sob o olhar dos seguintes elementos: contexto, autores, interesses, confiabilidade, natureza do texto e conceitos-chave ${ }^{11}$.

Esse estudo foi organizado nas seguintes etapas: seleção dos documentos oficiais por uma préleitura em que se buscou identificar as informações pertinentes ao objetivo do estudo; representação da amostra em formato de tabela, levando-se em consideração a proposta de cada documento; apresentação dos resultados identificando as orientações e recomendações presentes nos mesmos no que diz respeito ao aleitamento materno no momento de pandemia; e, por fim a análise descritiva e reflexiva dos dados.

\section{RESULTADOS E DISCUSSÃo}

Conforme processo de análise detalhado acima, os dados analisados delinearam quatro categorias: (1) Parturiente assintomática ou sem contato domiciliar com pessoa com síndrome gripal ou infecção respiratória comprovada por SARSCoV-2; (2) Parturiente sintomática ou com contato 
domiciliar com pessoa com síndrome gripal ou infecção respiratória comprovada por SARS-CoV-2; (3) Mulheres gestantes, parturientes e puérperas com diagnóstico de infecção pelo novo coronavírus ou em esclarecimento diagnóstico; (4) Condutas para a doação de leite materno aos bancos de leite humano e postos de coleta de leite humano no contexto da infecção Covid-19. A análise dos resultados foi realizada concomitante à apresentação dos dados.

Quadro 1 - Caracterização dos documentos analisados.

\begin{tabular}{|c|c|c|}
\hline Documento & Fonte/ano & Proposta \\
\hline $\begin{array}{l}\text { NOTA TÉCNICA } \\
\text { № 7/2020-DAPES/ } \\
\text { SAPS/MS }\end{array}$ & $\begin{array}{l}\text { Ministério da saúde. Secretaria } \\
\text { de Atenção Primária à Saúde. } \\
\text { Departamento de ações } \\
\text { programáticas estratégicas/2020 }\end{array}$ & $\begin{array}{l}\text { Avaliação de medida para o enfrentamento da situação } \\
\text { de emergência em saúde pública decorrente do } \\
\text { coronavírus (Covid-19), sobre questionamento da Rede } \\
\text { de Atenção à Saúde (RAS) respectivo à preservação } \\
\text { da amamentação em situação de risco iminente de } \\
\text { transmissão do respectivo vírus, em situações que } \\
\text { a mãe apresente sintomatologia compativel com } \\
\text { síndrome gripal. }\end{array}$ \\
\hline $\begin{array}{l}\text { NOTA TÉCNICA } \\
\text { № 8/2020-COCAM/ } \\
\text { CGCIVI/DAPES/ } \\
\text { SAPS/MS }\end{array}$ & $\begin{array}{l}\text { Ministério da saúde. Secretaria } \\
\text { de Atenção Primária à } \\
\text { Saúde } / 2020\end{array}$ & $\begin{array}{c}\text { Condutas para a doação de leite materno aos bancos } \\
\text { de leite humano e postos de coleta de leite humano } \\
\text { no contexto da infecção Covid-19 causada pelo novo } \\
\text { coronavírus (SARS-CoV-2). }\end{array}$ \\
\hline $\begin{array}{l}\text { NOTA TÉCNICA } \\
\text { №9/2020- DAPES/ } \\
\text { SAPS/MS }\end{array}$ & $\begin{array}{l}\text { Ministério da saúde. Secretaria } \\
\text { de Atenção Primária à } \\
\text { Saúde/2020 }\end{array}$ & $\begin{array}{l}\text { Trata-se de avaliação de medida para o enfrentamento } \\
\text { da situação de emergência em saúde pública no } \\
\text { contexto da infecção da Covid-19 causada pelo novo } \\
\text { coronavírus (SARS-CoV-2), sobre questionamento da } \\
\text { rede de atenção à saúde (RAS) respectivo à preservação } \\
\text { da amamentação em situação de risco iminente de } \\
\text { transmissão do respectivo vírus, em situações que } \\
\text { a mãe apresente sintomatologia compatível com } \\
\text { síndrome gripal. }\end{array}$ \\
\hline $\begin{array}{l}\text { NOTA TÉCNICA No } \\
\text { 10/2020-COCAM/ } \\
\text { CGCIVI/DAPES/ } \\
\text { SAPS/MS } \\
\end{array}$ & $\begin{array}{l}\text { Ministério da saúde. Secretaria } \\
\text { de Atenção Primária à } \\
\text { Saúde/2020 }\end{array}$ & $\begin{array}{l}\text { Atenção à saúde do recém-nascido no contexto da } \\
\text { infecção pelo novo coronavírus (SARS-CoV-2). }\end{array}$ \\
\hline Nota alerta & $\begin{array}{l}\text { Sociedade Brasileira de } \\
\text { Pediatria/2020 }\end{array}$ & $\begin{array}{l}\text { Prevenção e abordagem da infecção por Covid-19 em } \\
\text { mães e recém-nascidos, em hospitais-maternidades }\end{array}$ \\
\hline Nota alerta & $\begin{array}{l}\text { Sociedade Brasileira de } \\
\text { Pediatria/2020 }\end{array}$ & $\begin{array}{c}\text { Orientações a respeito da infecção pelo SARS-CoV-2 } \\
\text { (conhecida como Covid-19) em crianças }\end{array}$ \\
\hline Nota alerta & $\begin{array}{l}\text { Sociedade Brasileira de } \\
\text { Pediatria/2020 }\end{array}$ & O aleitamento materno nos tempos de Covid-19! \\
\hline
\end{tabular}

FONTE: Dados da pesquisa, 2020. 
A pactuação de uma política nacional de promoção, proteção e apoio ao aleitamento materno visa reforçar, adequar, ampliar e integrar intervenções e estratégias relacionadas ao mesmo, com vistas à sua consolidação como uma política de Estado, que induza e acelere a adesão à prática da amamentação e sua manutenção por tempo desejável, como preconizado pela Organização Mundial de Saúde (OMS), Fundação para as Ações Unidas para a Infância (UNICEF) e Ministério da Saúde (MS) ${ }^{12}$.

A prática hospitalar, durante anos, priorizou na assistência ao parto que a criança recém-nascida fosse afastada imediatamente da mãe para ser aquecida. Entretanto, este tipo de rotina vem sido transformada a partir de recomendações feitas por órgãos competentes como a UNICEF, a partir de evidências de benefícios a curto, longo e médio prazos para o binômio ${ }^{13}$.

Os documentos trouxeram as evidências existentes das práticas de cuidado da mulher e de recémnascidos no processo de amamentação nos primeiros dias após o parto, permitindo identificar as recomendações que contribuem para o aleitamento materno exclusivo no período intra-hospitalar, em tempos de pandemia por coronavírus, que, após o processo de análise, foram delineadas em quatro categorias:

\section{1- Parturiente assintomática ou sem contato domiciliar com pessoa com síndrome gripal ou infecção respiratória comprovada por SARS- CoV-2}

Com relação às orientações referentes ao aleitamento materno em sala de parto, os documentos apontam a necessidade de cautela, sendo necessário triar criteriosamente a história da gestante, a partir de seu cartão pré-natal, exames realizados e história clínica relatada. Se a parturiente for assintomática ou sem contato domiciliar com pessoa com síndrome gripal ou infecção respiratória comprovada por SARS-
CoV-2, orienta-se a manutenção da orientação da hora de ouro, mantendo a rotina de clampeamento em tempo oportuno do cordão umbilical ao nascimento, incentivando o contato pele a pele e o aleitamento materno na primeira hora de vida ${ }^{14}$.

\section{2- Parturiente sintomática ou com contato domiciliar com pessoa com síndrome gripal ou infecção respiratória comprovada por SARS- CoV-2}

Estudos recentes descreveram as características clínicas e laboratoriais da doença. Em relação às manifestações clínicas, a febre $(88,7 \%)$, tosse $(57,6 \%)$ e dispneia $(45,6 \%)$ foram as mais prevalentes. No tocante aos achados laboratoriais, diminuição da albumina $(75,8 \%)$, elevação da proteína C reativa $(58,3 \%)$ e da lactato desidrogenase (LDH) $(57,0 \%)$, linfopenia $(43,1 \%)$ e a alta taxa de sedimentação de eritrócitos (VHS) (41,8\%) foram os resultados mais prevalentes ${ }^{15}$. Nos exames de imagem, a radiografia de tórax mostra que o comprometimento pulmonar na pneumonia é predominantemente bilateral em $73 \%$ dos pacientes. Em relação aos pacientes, 20,3\% necessitaram de UTI. Desses 32,8\% tinham Síndrome do Desconforto Respiratório Agudo (SDRA); 13,0\% apresentavam lesão cardíaca aguda; 7,9\%, lesão renal aguda (IC95\% 1,8-14,0\%); 6,2\%, choque e 13,9\% tiveram resultados fatais ${ }^{16}$.

As formas de transmissão do coronavírus continuam sendo estudadas, sendo a disseminação pessoa a pessoa, através de gotículas salivares e respiratórias ou contato próximo e desprotegido com secreções ou excreções de um paciente infectado, o meio mais comum e conhecido até o momento. Outros fluidos corporais não estão claramente implicados na transmissão do novo coronavírus, mas se considera que o contato desprotegido com sangue, catarro, fezes, vômitos e urina pode oferecer risco de adoecimento $^{17,18}$.

Para a parturiente que for sintomática ou tenha 
contato domiciliar com pessoa acometida pelo SARS$\mathrm{CoV}-2$, as precauções consistem na manutenção de distância mínima de dois metros entre o leito materno e o berço do recém-nascido (RN), uso de máscara pela mãe sintomática durante o contato para cuidados e durante toda a amamentação, precedida pela higienização adequada das mãos antes e após o contato com a criança ${ }^{14}$. Deverá ser assegurado a esta mãe condutas acolhedoras, deixando claro os benefícios do aleitamento materno ${ }^{19}$.

\section{3- Mulheres - gestantes, parturientes e} puérperas com diagnóstico de infecção pelo novo coronavírus ou em esclarecimento diagnóstico

Nesse caso, a amamentação pode ser mantida, assegurando a autonomia da mulher, com as precauções necessárias para evitar contaminação do RN: uso de máscara cirúrgica para amamentar, distância de dois metros entre leito materno e o berço nos intervalos de mamadas, e higienização adequada das mãos antes e após os cuidados com o $\mathrm{RN}^{14}$.

As máscaras faciais, quando adaptadas adequadamente, interrompem efetivamente a dispersão das partículas expelidas por meio da tosse ou espirro, impedindo a transmissão de doenças respiratórias. Mesmo máscaras que não se adaptam perfeitamente, bem como máscaras de fabricação caseira, embora com desempenho inferior às máscaras cirúrgicas e N95, são capazes de reter partículas e vírus transportados pelo ar, de modo que esses não alcancem pessoas próximas ${ }^{20}$.

Estudo recente concluiu que o uso de máscaras cirúrgicas por pacientes com doença aguda provocada por coronavírus é eficaz na redução do risco de transmissão, sendo uma prática promissora para diminuir a velocidade de disseminação de doenças respiratórias por reduzir a transmissão do aerossol, quando nos casos de contato com pessoas acometidas de Covid-19 e com sintomas respiratórios brandos, devendo seu uso acontecer desde a chegada ao serviço de saúde, durante o isolamento e na circulação dentro do serviço (transporte de uma área/ setor para outro) devendo-se evitar ao máximo tocar a máscara, olhos, boca e face ${ }^{21}$.

\section{4- Condutas para a doação de leite materno aos bancos de leite humano e postos de coleta de leite humano no contexto da infecção Covid-19}

O Banco de Leite Humano tem se configurado como um dos mais importantes elementos estratégicos da política pública em favor da amamentação. Sempre vinculado a uma Unidade Hospitalar, tem por objetivo promover, proteger e apoiar o aleitamento materno e execução de atividades de coleta da produção láctea da nutriz, seleção, classificação, processamento, controle de qualidade e distribuição ${ }^{22}$. A Rede Brasileira de Banco de Leite Humano é considerada a maior e mais complexa do mundo pela OMS. Dentre os 292 bancos de leite humano existentes no mundo, 72,9\% deles estão no Brasil ${ }^{23}$.

No tocante ao aleitamento materno, estes bancos de leite surgem a partir de novos saberes, práticas e soluções frente às causas do desmame precoce, orientado pela filosofia do assistir, adaptando cenários de mudança, representando uma estratégia importante para a amamentação e para a manutenção do aleitamento dos bebês que não podem mamar no peito, repercutindo positivamente na redução da mortalidade desses públicos-alvo ${ }^{24}$.

No cenário da pandemia pelo Covid-19, fez-se necessário orientar os serviços de saúde que realizam a coleta, armazenamento e processamento de leite humano, bem como os profissionais de saúde que atuam nos procedimentos de manejo de leite humano e no atendimento às famílias na fase de lactação.

Para as lactantes assintomáticas ou sem contato domiciliar com pessoa com síndrome gripal ou infecção respiratória comprovada por SARS-CoV-2, mantém-se a recomendação de doação de leite materno, desde que sejam adotadas medidas de 
precaução sobre risco de síndrome gripal, conforme protocolos existentes do Ministério da Saúde ${ }^{25}$.

Entretanto, é contraindicada a doação por mulheres com sintomas compatíveis com síndrome gripal, infecção respiratória ou confirmação de caso da Covid-19. A contraindicação é estendida a mulheres contatos domiciliares de casos com síndrome gripal ou caso confirmado de Covid-1925.

Para a coleta do leite materno, ficam válidos os critérios e recomendações da Rede Brasileira de Bancos de Leite e da Agência Nacional de Vigilância Sanitária (Anvisa). É importante que as unidades gestoras de saúde possam ofertar, além das redes de atenção que já existem, alternativas que assegurem a manutenção das doações, garantindo o acesso às informações e a disponibilidade de leite humano, estimulando a manutenção da lactação exclusiva nos primeiros seis meses de vida.

\section{CONCLUSÕES}

A elaboração de Notas Técnicas pelo Ministério da Saúde, órgão que rege as condutas de saúde no País, frente à pandemia pelo Covid-19, tem como finalidade promover orientações que garantam a saúde da mulher e da criança, mediante a determinação de fluxos assistenciais e práticas clínicas a partir de informações técnicas baseadas nas melhores evidências até o momento.

Quanto às orientações na promoção do aleitamento materno frente à pandemia pelo coronavírus, em sala de parto é necessário triar criteriosamente a história da gestante, a partir de seu cartão prénatal, exames realizados e história clínica relatada. Se a parturiente for assintomática ou sem contato domiciliar com pessoa com síndrome gripal ou infecção respiratória comprovada por SARS-CoV-2, mantém-se a orientação da hora de ouro, a rotina de clampeamento em tempo oportuno do cordão umbilical ao nascimento, incentivando o contato pele a pele e o aleitamento materno na primeira hora de vida

Para a parturiente que for sintomática ou tenha contato domiciliar com pessoa acometida pelo SARSCoV-2, as precauções consistem na manutenção de distância mínima de dois metros entre o leito materno e o berço do recém-nascido, uso de máscara pela mãe sintomática durante o contato para cuidados e durante toda a amamentação, precedida pela higienização adequada das mãos antes e após o contato com a criança

No tocante à doação de leite materno, as lactantes assintomáticas ou sem contato domiciliar com pessoa com síndrome gripal ou infecção respiratória comprovada por SARS-CoV-2, podem doar leite materno, desde que sejam adotadas medidas de precaução sobre risco de síndrome gripal, conforme protocolos existentes do Ministério da Saúde.

Entretanto, é contraindicada a doação por mulheres com sintomas compatíveis com síndrome gripal, infecção respiratória ou confirmação de caso da Covid-19. A contraindicação é estendida a mulheres com contatos domiciliares de casos com síndrome gripal ou caso confirmado de Covid-19.

Estas publicações são um importante instrumento de apoio aos profissionais, seja na realização das práticas envolvendo o parto, puerpério, o aleitamento materno e os bancos de leite humano, ou ainda na vigilância destes.

Devido à possibilidade de novas descobertas que possam ser feitas e da atualização na literatura científica num momento de pandemia, as notas elaboradas podem ser revistas, atualizadas ou mesmo revogadas, em favor das melhores práticas sanitárias e do cuidado centrado nas pessoas e suas necessidades, sendo imprescindível que os profissionais de saúde estejam mantendo constante e pertinente atualização sobre a temática do cuidado a gestante, puérpera e aleitamento materno na pandemia existente.

As atualizações frequentes das notas técnicas configuraram a principal limitação desse estudo. 


\section{REFERÊNCIAS}

1. Ministério da Saúde (BR). Protocolo de manejo clínico do coronavírus (Covid-19) na atenção primária à saúde. 4 ed. Secretaria de Atenção Primária à Saúde (SAPS), Brasília; 2020. [cited 2020 Apr 25]. Available from: https://saude.gov. br/images/pdf/2020/marco/23/20200323-ProtocoloManejover04.pdf.

2. Carbinatto B. Coronavírus no Brasil: cinco perguntas para entender a chegada do vírus. Revista Superinteressante. [revista na internet] 2020 abril. [cited 2020 Apr 5]. Available from: https://super.abril.com.br/saude/coronavirus-nobrasil-5-perguntas-para-entender-a-chegada-do-virus-aopais/

3. Painel Coronavírus no Brasil [homepage na internet]. Painel de casos de doença pelo coronavírus 2019 (COVID-19) no Brasil pelo Ministério da Saúde. 2020. [cited 2020 May 23]. Available from: http:www.covid.saude. gov.br

4. Wang W, Tang J, Wei F. Updated understanding of the outbreak of 2019 novel coronavirus (2019-nCoV) in Wuhan, China. J Med Virol. 2020; [cited 2020 Apr 25]. 92(4):441-447. Available from: https://doi.org/10.1002/jmv.25689.

5. Ministério da Saúde (BR). Protocolo de manejo clínico da Covid-19 na Atenção Especializada. Secretaria de Atenção Especializada à Saúde, Departamento de Atenção Hospitalar, Domiciliar e de Urgência; Brasília - 1. ed. rev 2020. [cited 2020 Apr 24]. Available from: http://bvsms. saude.gov.br/bvs/publicacoes/manejo_clinico_covid-19 atencao_especializada.pdf ISBN 978-85-334-2766-

6. Ministério da Saúde (BR). Saúde da criança: aleitamento materno e alimentação complementar. 2 ed. Secretaria de Atenção à Saúde, Departamento de Atenção Básica, Brasília; 2015. [cited 2020 Apr 26]. Available from: https:// bvsms.saude.gov.br/bvs/publicacoes/saude_crianca_ aleitamento_materno_cab23.pdf

7. Neto HFDS, Almeida JAVD, Costa MSDC, Hazboun ND, \& Leite VMC. (2017). A importância do aleitamento materno exclusivo: um relato de experiência. Revista Extensão \& Sociedade. 2017 [cited 2020 Apr 25] 6(2):5966. Available from: http://www.repositorio.ufc.br/handle/ riufc/35627

8. Matos TA, Souza MSD, Santos EKAD, Velho MB, Seibert ERC, \& Martins NM. Precocious skin-to-skin contact between mother and child: meanings to mothers and contributions for nursing. Rev Bras Enferm. 2010 [cited 2020 Apr 28] 63(6):998-1004. Available from: https://doi. org/10.1590/S0034-71672010000600020

9. Silveira DT, Córdova FP. A pesquisa científica. In: Gerhardt TE, Silveira DT. Métodos de Pesquisa. Porto Alegre: UFRGS, 2009, p. $31-41$

10. Gil AC. Como elaborar projetos de pesquisa. São Paulo: Atlas, 2002. p. 87 - 91.

11. Cechinel A, Fontana SAP, Giustina KPD, Pereira AS, Prado SS. Estudo/Análise documental: uma revisão teórica e metodológica. Criar Educação, 5(1): 1-7, 2016. [cited 2020 may 06]. Available from: http://dx.doi.org/10.18616/ ce.v5i1.2446.

12. Ministério da Saúde (BR). Bases para a discussão da Política Nacional de Promoção, Proteção e Apoio ao Aleitamento Materno. Secretaria de Atenção Primária à Saúde (SAPS), Departamento de Ações Programáticas Estratégicas. Brasília; 2020. [cited 2020 Apr 28]. Available from: http://bvsms.saude.gov.br/bvs/publicacoes/bases_ discussao_politica_aleitamento_materno.pdf

13. Victora CG, Bahl R, Barros AJ. Breastfeeding in the 21st century: epidemiology, mechanisms, and lifelong effect. Lancet. 2016 [cited 2020 Apr 24] 387:475-490. Available from: https://doi.org/10.1016/S0140-6736(15)01024-7

14 Ministério da Saúde (BR). Nota técnica $\mathrm{N}^{\circ} 10$. Atenção à saúde do recém-nascido no contexto da infecção pelo novo coronavírus (SARS-CoV-2). Secretaria de Atenção Primária à Saúde (SAPS), Brasília; 2020. [cited 2020 Apr 25]. Available from: https://portaldeboaspraticas.iff.fiocruz. br/atencao-recem-nascido/atenc\%CC\%A7a\%CC\%83o-asaude-do-recem-nascido-no-contexto-da-infeccao-pelonovo-coronavirus-sars-cov- $2 /$

15. Rodriguez-Morales AJ, Cardona-Ospina JA, GutiérrezOcampo E, Villamizar-Peña R, Holguin-Rivera Y, EscaleraAntezana JP, et al. Clinical, laboratory and imaging features of COVID-19: A systematic review and metaanalysis. Travel Medicine and Infectious Disease. 2020 [cited 2020 May 25]:101623. Available from: doi:10.1016/j. tmaid.2020.101623

16. Ministério da Saúde (BR). Diretrizes para diagnóstico e tratamento da COVID-19. Secretaria de Ciência, Tecnologia, Inovação e Insumos Estratégicos em Saúde - SCTIE, Brasília; 2020. [cited 2020 May 25]. Available 
from: https://sbim.org.br/images/files/notas-tecnicas/ddtcovid-19-200407.pdf

17. Del Rio, C., \& Malani, P. N. (2020). 2019 Novel coronavirus-important information for clinicians. Jama, [cited 2020 May 23]; 323(11):1039-40. Avaliable from: https:// doi.org/10.1001/jama.2020.1490.

18. Centers for Disease Control and Prevention. Interim U.S. guidance for risk assessment and public health management of healthcare personnel with potential exposure in a healthcare setting to patients with Coronavirus Disease (COVID-19). 2020 [acessed 2020 may 22]. Available from: https://www.cdc.gov/coronavirus/2019ncov/hcp/guidance-risk-assesment-hcp.html

19. Ministério da Saúde (BR). Nota técnica $N^{\circ} 09$. Avaliação de medida para o enfrentamento da situação de emergência em saúde pública no contexto da infecção da Covid-19 causada pelo novo coronavírus (SARS-CoV-2), sobre questionamento da rede de atenção à saúde (RAS) respectivo à preservação da amamentação em situação de risco iminente de transmissão do respectivo vírus, em situações que a mãe apresente sintomatologia compatível com síndrome gripal. Secretaria de Atenção Primária à Saúde (SAPS), Brasília; 2020. [cited 2020 Apr 22]. Available from: http://189.28.128.100/dab/docs/portaldab/documentos/ecnicaamamentacao92020DAPESSAPSMS03abr2020COVID-19.pdf

20. Lai ACK; Poon CKM; Cheung ACT. Effectiveness of facemasks to reduce exposure hazards for airborne infections among general populations. Journal of the Royal Society Interface, 2012 [cited 2020 Apr 25] 9(70):938-948. Available from: https://doi.org/10.1098/rsif.2011.0537

21. Leung NHL, Chu DKW, Shiu EYC et al. Derramamento do vírus respiratório na respiração exalada e eficácia das máscaras faciais. Nat Med 26, 676-680 (2020). https://doi. org/10.1038/s41591-020-0843-2

22. Oliveira ND, da Silva EB, de Lima Moura R, Dantas ENDA, de Sousa JTA, da Silva CP, \& Jerônimo HMÂ. Importância do banco de leite humano nas unidades de alimentação e nutrição: uma revisão de literatura. International Journal of
Nutrology, 2018 [cited 2020 Apr 26] 11(S01):453. Available from: https://doi.org/10.1055/s-0038-1674750

23. Ministério da Saúde (BR). Portal da Saúde. [homepage da internet] Brasil tem o maior número de doadoras de leite humano do mundo. 2020. [cited 2020 Apr 09]. Available from: http://portalsaude.saude.gov.br/index.php/oministerio/ principal/secretarias/sas/sasnoticias/22451-brasil-tem-omaior-numero-de-doadoras-de-leite-humano-do-mundo

24. Luna FDT, Oliveira JDL, de Mello Silva LR. Banco de leite humano e Estratégia Saúde da Família: parceria em favor da vida. Rev. Bras. Med. Fam. Comunidade, 2014 [cited 2020 Apr 25] 9(33): 358-364. Available from: https:// doi.org/10.5712/rbmfc9(33)824

25. Ministério da Saúde (BR). Condutas para a doação de leite materno aos bancos de leite humano e postos de coleta de leite humano no contexto da infecção Covid 19 causada pelo novo coronavírus (SARS-CoV-2). Secretaria de Atenção Primária à Saúde(SAPS), Brasília;2020. [cited2020 Apr 23]. Availablefrom: http://189.28.128.100/dab/docs/portaldab/documentos/ notatecnicadoacaoleite82020COCAMCGCIVIDAPESSAPSMS03abr2020COVID-19.pdf 